www.nature.com/jhg

\title{
Association between dopaminergic genes (SLC6A3 and DRD2) and stuttering among Han Chinese
}

\author{
Jie Lan ${ }^{1,4}$, Manshu Song ${ }^{2,4}$, Chunhui Pan ${ }^{1}$, Guoqing Zhuang ${ }^{1}$, Youxin Wang ${ }^{2}$, Wenzhan Ma ${ }^{1}$, Qiaoyun Chu ${ }^{1}$, \\ Qingxuan Lai ${ }^{1}$, Feng $\mathrm{Xu}^{1}$, Yanli $\mathrm{Li}^{1}$, Lixin $\mathrm{Liu}^{1}$ and Wei Wang ${ }^{1,2,3}$
}

\begin{abstract}
Normal function of the dopaminergic system is necessary for speech fluency. There was evidence that the activities of dopamine transporter (DAT) and dopamine D2 receptor (DRD2) could be altered in people with speech disfluency. This study aims to ascertain the possible correlation between two dopaminergic genes (SLC6A3 and DRD2) and disorder of speech fluency, and to determine the allelic frequencies of the five single-nucleotide polymorphisms (SNPs) (rs2617604, rs28364997, rs28364998 in SLC6A3 and rs6275, rs6277 in DRD2) among Han Chinese patients with this disorder. A sample of 112 patients with speech disfluency and 112 gender-matched controls were included in this case-control study. The results show that the presence of C allele at rs6277 in DRD2 gene is associated with increased susceptibility to the disorder, whereas T allele is protective. Haplotype $939 \mathrm{~T} / 957 \mathrm{~T}$ is also a protective factor.
\end{abstract}

Journal of Human Genetics (2009) 54, 457-460; doi:10.1038/jhg.2009.60; published online 10 July 2009

Keywords: case-control study; dopamine D2 receptor; dopamine transporter; disorder of fluent speech; Han Chinese; SNPs

\section{INTRODUCTION}

Language is a particular type of human cognitive function that can be divided into 'language' and 'speech.' ${ }^{1}$ Speech production is a process that transforms conceptual ideas within the brain into specific language form and expresses it vocally. ${ }^{2}$ Fluent speech depends on the balance of the three steps: conceptualization, formulation and articulation, ${ }^{3}$ but the detailed mechanism has not been fully understood yet. Stuttering has been used as a model of speech disfluency to understand the mechanism of speech production. ${ }^{4}$ It is characterized by involuntary syllable repetitions, syllable prolongations or interruptions (blocks) in a smooth flow of speech. Developmental stuttering begins during the period of rapid speech and language development in childhood and is characterized by a disturbance in the normal fluency and time patterning of speech. ${ }^{5}$

Dopamine has a major role in fine motor movements, and speech requires the coordination of many small muscles. ${ }^{6}$ The dopamine excess theory of stuttering suggests that stuttering may be related to excess levels of the neurotransmitter in the brain. ${ }^{7-9}$ Further evidence for the theory came from attempts at treating stuttering with antipsychotic medications that block dopamine in the brain. ${ }^{10-13}$ A study to evaluate the efficacy of risperidone in the treatment of developmental stuttering showed that a significant decrease of stuttering severity occurred in the risperidone treatment group than in that of the placebo group. ${ }^{14}$ The risperidone research supports dopamine having a regulatory role in stuttering.
The prevalence of children who stutter and have attention deficit/ hyperactivity disorder (ADHD) ranges from 4 to $26 \%$. About $10-20 \%$ of children who stutter might show ADHD. ${ }^{15}$ The data suggest that stuttering and ADHD may have some common pathology. One nonsynonymous single-nucleotide polymorphism (SNP) in SLC6A3, rs28364997 (A559V), was identified in screening children diagnosed with ADHD, and two affected male siblings were heterozygous for the A559V allele. ${ }^{16}$ Human embryonic kidney cells transfected with the A559V dopamine transporter (DAT) (hDAT A559V cells) displays increased DA efflux when compared with cells transfected with DAT (hDAT cells), suggesting that the A559V variant is associated with a DAT-mediated DA leak that may underlie the ADHD phenotype. ${ }^{17}$ The other SNP in DRD2 (rs6277) shows that carriers of the CC genotype, compared with carriers of the CT/TT genotypes, achieve significantly poorer executive functioning. It has been found that a lower dopamine D2 receptor (DRD2) binding was associated with the CC genotype of rs6277 (SNP of the DRD2 gene). This led to an increased density of dopamine in the synaptic cleft in in vivo studies. ${ }^{18}$ However, to date, no study has examined the association between rs2617604, rs28364997, rs28364998, rs6275, rs6277 polymorphisms and speech disfluency.

This study aims to examine the polymorphisms (rs2617604, rs28364997, rs28364998 in SLC6A3 gene and rs6275, rs6277 in $D R D 2$ gene) and their haplotypes in a sample of disfluent speech and controls. We also aim to test whether the two dopaminergic genes

${ }^{1}$ Department of Genetics, College of Life Sciences, Graduate University of Chinese Academy of Sciences, Beijing, PR China; ${ }^{2}$ School of Public Health and Family Medicine, Capital Medical University, Beijing, PR China and ${ }^{3}$ School of Public Health, Curtin University, Perth, WA, Australia

${ }^{4}$ These authors contributed equally to this work.

Correspondence: Professor W Wang, Department of Genetics, College of Life Sciences, Graduate University of Chinese Academy of Sciences, No 19A Yue Quan Road, Beijing 100049, PR China. E-mails: wei6014@yahoo.com or wei6014@gucas.ac.cn

Received 11 April 2009; revised 18 June 2009; accepted 19 June 2009; published online 10 July 2009 
Table 1 Primers and fragmental length used in sample preparation for single-nucleotide polymorphism (SNP) analysis

\begin{tabular}{|c|c|c|c|c|}
\hline & Name & Primer sequence & $\begin{array}{l}\text { Length } \\
\text { (bp) }\end{array}$ & SNP \\
\hline$\rightarrow \mathrm{PCR}$ & DAT $2 \mathrm{~F} 1$ & TATTGGCTGAAGACCAAGAGG & & \\
\hline$\rightarrow$ Sequencing & DAT 2S1 & GGAGCAGAACGGAGTG & & \\
\hline$\rightarrow \mathrm{PCR}$ & DAT $13 F 1$ & TATCTGCTGGTTGCAGTTCG & & \\
\hline - PCR & DAT 13R1 & CAGAACTTGTAGGCCGCATAGA & 159 & rs28364997 \\
\hline$\sim \mathrm{PCR}$ & $\mathrm{DRD}_{2} 6 \mathrm{R} 2$ & GCAATCTTGGGGTGGTCTTT & 113 & rs 6275 \\
\hline - Sequencing & $\mathrm{DRD}_{2} 6 \mathrm{~S} 2$ & TGGTTTGGCGGGGCT & & rs6277 \\
\hline
\end{tabular}

$\sigma, \sim$ biotin labeled.

are involved in the genetic susceptibility to disorders of fluent speech in a Han Chinese population who speak Mandarin, a hieroglyphic language with many differences from English, an alphabetic language.

\section{MATERIALS AND METHODS}

Study participants

This study included 112 stutterers (the patients) and 112 non-stutterers (the controls). The stutterers were recruited from two Rectification Centres of Stuttering (Beijing Stuttering Correction Centre and Dingguagua Stutter Club in Xi'an) and one hospital (Beijing Charity Hospital). Participants suffering from neurological or other physical diseases were excluded from the study. There were 13 females and 99 males in the patient group and their mean age was 23.53 years (s.d.: 4.99). Chinese Fluency Interview, an instrument specifically modified from the Fluency Interview to be applicable to Chinese, ${ }^{19}$ was used for the assessment of stuttering. The total number of times of stuttering and the number of stuttering episodes per minute were calculated. More than 3 times per minute was diagnosed as stuttering.

The non-stutterers were recruited from college students who volunteered to participate. Participants with a past history or family history of fluency disorders, severe neurological or other physical diseases were excluded. Among the enrolled control participants, 112 participants were selected to match the sex ratio with the patient group ( 13 females and 99 males). Their mean age was 25.23 years (s.d.: 3.34 ).

All participants are native Han Chinese. Informed consents were obtained beforehand, and ethical approval was obtained from the Ethical Committee, Graduate University of the Chinese Academy of Sciences.

\section{Genotyping}

Genomic DNA was obtained from peripheral blood leukocytes using standard methods. A combined approach of PCR and pyrosequencing methods was used for genotyping. The fragments that contained the SNPs in DRD2 were amplified with biotinylated forward primers and non-biotinylated reverse primers, whereas the fragments that contained the SNPs in SLC6A3 gene were amplified with biotinylated reverse primers and non-biotinylated forward primers, respectively (Table 1). PCR amplification was carried out in a $50 \mu \mathrm{l}$ volume containing $1 \mu \mathrm{l}(10 \mathrm{ng})$ genomic DNA, $5 \mu 110 \times$ PCR buffer, $200 \mu \mathrm{M}$ dNTP, $200 \mathrm{~nm}$ primer of each and $0.5 \mu \mathrm{l}(2.5 \mathrm{U})$ Taq DNA polymerase. After $5 \mathrm{~min}$ of pre-denaturation at $95^{\circ} \mathrm{C}, 50$ thermal cycles were repeated, $15 \mathrm{~s}$ at $95^{\circ} \mathrm{C}, 30 \mathrm{~s}$ at annealing temperature, and $15 \mathrm{~s}$ at $72^{\circ} \mathrm{C}$, the PCR tubes were then incubated for $5 \mathrm{~min}$ at $72^{\circ} \mathrm{C}$ for final extension. The genotypes of the PCR products were determined by pyrosequencing using a standard protocol. Briefly, following strand separation, the biotinylated PCR product was immobilized on streptavidin-coated beads (Streptavidin Sepharose High Performance; Amersham Biosciences, Uppsala, Sweden). The beads were transferred to a filter plate, and the liquid was removed by vacuum filtration (Multiscreen Resist Vacuum Manifold; Millipore, Billerica, MA, USA). The DNA strands were separated in a denaturation solution $\left(0.2 \mathrm{moll}^{-1} \mathrm{NaOH}\right)$ for $5 \mathrm{~s}$. The immobilized template was washed in $10 \mathrm{nmoll}^{-1}$ Tris acetate ( $\mathrm{pH}$ 7.6), transferred to a
Table 2 Details about selected single-nucleotide polymorphisms (SNPs)

\begin{tabular}{|c|c|c|c|c|c|}
\hline Gene & $\begin{array}{l}\text { Chromosome } \\
\text { location }\end{array}$ & refSNP ID & SNP & Protein residue & Region \\
\hline & & rs2617604 & $124 \mathrm{C}>\mathrm{T}$ & Leu42Phe & Exon-2 \\
\hline \multirow[t]{2}{*}{ SLC6A3 } & $5 p 15.3$ & rs28364997 & $1676 \mathrm{C}>\mathrm{T}$ & Ala559Val & Exon-13 \\
\hline & & rs28364998 & $1692 \mathrm{C}>\mathrm{T}$ & Ile564lle & Exon-13 \\
\hline \multirow[t]{2}{*}{ DRD2 } & $11 q 23$ & rs6275 & 939T $>C$ & $\mathrm{His} 313 \mathrm{His}$ & Exon-7 \\
\hline & & rs6277 & $957 \mathrm{C}>\mathrm{T}$ & Pro319Pro & Exon-7 \\
\hline
\end{tabular}

PSQ 96 plate, and resuspended in annealing buffer $\left(20 \mathrm{mmoll}^{-1}\right.$ Tris acetate ( $\mathrm{pH}$ 7.6)) that contained sequencing primers (Table 1). The resulting mixture was analyzed on a PSQ 96MA Pyrosequencer (Pyrosequencing AB; Biotage, Uppsala, Sweden) using the multiplex programing function.

\section{Statistical methods}

The allelic and genotypic frequencies, odds ratios (OR), haplotypes construction and haplotype frequencies were analyzed using the online SHEsis software $^{20}$ (http://analysis.bio-x.cn/myAnalysis.php). The differences in the frequencies of various alleles and genotypes between patients and controls were performed by $\chi^{2}$-test with Yates' correction, and Fisher's exact test was applied to the loci with a small number of alleles or genotypes (less than 5). $P<0.05$ was considered significant. Each polymorphism was evaluated for the Hardy-Weinberg equilibrium by a $\chi^{2}$-test in both patients and controls. Values of $P>0.05$ were defined as nonsignificant deviations from Hardy-Weinberg equilibrium. Statistical power was calculated by the Genetic Power Calculator (http://pngu.mgh.harvard.edu/ purcell/gpc/\#cc_ins).

\section{RESULTS}

SNPs in SLC6A3 and DRD2 genes among the Han Chinese population

A total of five SNPs (Table 2) were detected in the patient and control groups combined. Among them, two SNPs (rs2617604 and rs28364997) are non-synonymous variants leading to amino-acid substitutions (Leu42Phe and Ala559Val).

\section{The allelic and genotypic frequencies of the SNPs at SLC6A3 and DRD2 genes}

Analysis showed that the patients and controls were in the HardyWeinberg equilibrium at rs2617604 ( $P=0.962$ and 0.962$)$, rs28364997 ( $P=1.000$ and 1.000), rs28364998 $(P=0.886$ and 0.771$)$, rs6275 $(P=0.204$ and 0.124$)$ and rs6277 $(P=0.695$ and 0.280$)$. 
Table 3 Frequencies of genotype and allele of SLC6A3 and DRD2 gene polymorphisms in disfluent speech patients and controls

\begin{tabular}{|c|c|c|c|c|c|}
\hline Polymorphism & Patients, n (\%) & Controls, n (\%) & $\chi^{2}$ & $P$ & OR $(95 \% \mathrm{Cl})$ \\
\hline \multicolumn{6}{|l|}{ rs2617604 } \\
\hline \multicolumn{6}{|l|}{ Alleles } \\
\hline $\mathrm{T}$ & $1(0.4)$ & $1(0.4)$ & & & \\
\hline \multicolumn{6}{|l|}{ Genotypes } \\
\hline $\mathrm{CC}$ & $111(99.1)$ & $111(99.1)$ & & $1.000^{\mathrm{a}}$ & $1.000(0.062-16.189)$ \\
\hline \multicolumn{6}{|l|}{ rs28364998 } \\
\hline \multicolumn{6}{|l|}{ Alleles } \\
\hline$c$ & $221(98.7)$ & $218(97.3)$ & & $0.5034^{a}$ & $2.028(0.501-8.210)$ \\
\hline $\mathrm{T}$ & $3(1.3)$ & $6(2.7)$ & & & \\
\hline \multicolumn{6}{|l|}{ Genotypes } \\
\hline $\mathrm{CC}$ & 109 (97.3) & $106(94.6)$ & & $0.4988^{a}$ & $2.057(0.501-8.436)$ \\
\hline $\mathrm{T}$ & $124(55.4)$ & $127(56.7)$ & & & \\
\hline \multicolumn{6}{|l|}{ Genotypes } \\
\hline CC & $19(17.0)$ & $17(15.2)$ & 0.135 & 0.716 & \\
\hline СT & $62(55.4)$ & $63(56.2)$ & & 0.893 & \\
\hline $\mathrm{TT}$ & $31(27.7)$ & $32(28.6)$ & & 0.882 & \\
\hline \multicolumn{6}{|l|}{$r s 6277$} \\
\hline \multicolumn{6}{|l|}{ Alleles } \\
\hline c & $216(96.4)$ & $196(87.5)$ & 12.082 & 0.001 & $3.857(1.717-8.663)$ \\
\hline $\mathrm{T}$ & $8(3.6)$ & $28(12.5)$ & & & \\
\hline \multicolumn{6}{|l|}{ Genotypes } \\
\hline $\mathrm{CC}$ & 104 (92.9) & $87(77.7)$ & & $0.0019^{a}$ & \\
\hline
\end{tabular}

Statistically significant difference, $P<0.05$; statistically nonsignificant, $P>0.05$.

aFisher's Exact Test.

Among the five SNPs investigated, rs28364997 did not show any polymorphism either in the patient group or in the control group. Therefore, rs28364997 was excluded from subsequent analysis.

There was no significant difference in the allelic and genotypic frequencies of the rs2617604, rs28364998 and rs6275 polymorphisms between the patients and controls $(P>0.05)$, whereas the patient group showed significant variation at the rs6277 site (Table 3 ). The frequency of $\mathrm{C}$ alleles at the rs6277 site was significantly higher, and that of $\mathrm{T}$ alleles was significantly lower, in the patient group than the controls (96.4 vs 87.5 and 3.6 vs $12.5 \%$, respectively, $P=0.001$ ). The results showed that rs6277 in the DRD2 gene had the highest association with disorders of fluent speech. $\mathrm{C}$ allele was a risk factor to this disorder $(P=0.001 ; \mathrm{OR}=3.857,95 \%$ confidence interval 1.717-8.663).

\section{Haplotype analysis of the SNPs in SLC6A3 and DRD2 genes}

The frequencies of various haplotypes constructed by rs 2617604 and rs28364998 in the SLC6A3 gene and rs6275 and rs6277 in the DRD2 gene were determined by SHEsis software. The haplotypes with frequency $<0.03$ in both patients and controls were ignored during the analysis. The five possible haplotype frequencies are shown in Table 4.

There was no significant difference in the haplotype frequency of SLC6A3 between the patient and control groups. Among the various possible haplotypes of the DRD2 gene $(n=4)$, the frequency of the
939T/957T haplotype was significantly lower in the patients than in the controls $(0.0$ vs $4.9 \%, P=0.001)$. The OR of the $939 \mathrm{~T} / 957 \mathrm{~T}$ haplotype is 0.001 ( $95 \%$ confidence interval $0.000-0.010$ ), with significant differences between patients and controls $(P=0.001)$.

\section{DISCUSSION}

Studies concerning the nature of language recognition have produced an extensive amount of data over the years, but the biological mechanisms of language recognition, production and speech disfluency problems are still not clear. To our knowledge, this is the first attempt to evaluate the association between the SNPs and haplotypes of the SLC6A3 and DRD2 genes and disorders of fluent speech in the Chinese Han population. Our finding lends direct support to the hypothesis that the dopaminergic system is involved in stuttering, and suggests a potential genetic risk factor for stutterers of the Chinese Han population.

Hans-Georg Bosshardt ${ }^{21}$ analyzed how silent reading and word memorization may affect the fluency of concurrently repeated words. It has been found that the speech of stutterers is more sensitive to interference from concurrently performed cognitive activity. ${ }^{21}$ Recent animal studies indicate that changes in DRD2 expression in the striatum modify prefrontal dopaminergic function through the changing dopamine level, its turnover rate and activation of D1 receptors, 
Table 4 Frequency of haplotypes of SLC6A3 and DRD2 gene polymorphisms in disfluent speech patients and controls

\begin{tabular}{|c|c|c|c|c|c|}
\hline Haplotypes & Patients (\%) & Controls (\%) & $\chi^{2}$ & $P$ & OR $(95 \%$ Cl) \\
\hline \multicolumn{6}{|l|}{$S L C 6 A 3$} \\
\hline $124 C / 1692 C$ & $220.00(98.2)$ & $217.00(96.9)$ & 0.000 & 1.000 & - \\
\hline $124 \mathrm{~T} / 1692 \mathrm{C}$ & $1.00(0.4)$ & $1.00(0.4)$ & - & - & - \\
\hline \multicolumn{6}{|l|}{ DRD2 } \\
\hline 939T/957C & $123.99(55.4)$ & $115.97(51.8)$ & 0.577 & 0.447 & $1.155(0.796-1.675)$ \\
\hline 939T/957T & $0.01(0.0)$ & $11.03(4.9)$ & 10.371 & 0.001 & $0.001(0.000-0.010)$ \\
\hline
\end{tabular}

Abbreviations: $\mathrm{Cl}$, confidence interval; OR, odds ratio.

Statistically significant difference, $P<0.05$; statistically nonsignificant, $P>0.05$.

all of which are crucial in cognitive functions. ${ }^{22}$ This study shows that the DRD2 polymorphism is associated with the susceptibility to disorders of fluent speech in the Han Chinese. C allele at rs6277 is the risk factor to the disorder $(P=0.001 ; \mathrm{OR}=3.857,95 \%$ confidence interval 1.717-8.663). Although the mutation rs6277 in DRD2 does not change the amino acid (Pro 319 Pro), studies in vivo have associated the CC genotype of rs6277 to a lower striatal DRD2 binding. ${ }^{23}$ Then most of the DA in the synaptic cleft would be reuptaken by DAT resulting in an overactivity of the presynaptic dopamine system in the brain. In this study, patients have a significantly higher CC genotype $(P=0.001)$ and lower CT genotype $(P=0.006)$ when compared with the controls, which is compatible with an earlier result in which stuttering patients showed a large increase in dopamine activation in the presynaptic area. ${ }^{9}$ Among the haplotypes of the DRD2 gene, the frequency of 939T/957T is significantly lower in the patient group $(P=0.001 ; \mathrm{OR}=0.001,95 \%$ confidence interval $0.000-0.010)$, indicating its protective effect on disorders of fluent speech in the Han Chinese. The results showed that the highest association is observed between rs6277 in the DRD2 gene and disorders of fluent speech. In addition, the statistical power was calculated by the Genetic Power Calculator and it equals $80.37 \%$ (high-risk allele frequency A equals 0.9196; prevalence equals 1\%; genotypic relative risk AA equals 14.88).

There were no significant associations detected between the other three SNPs (rs2617604, rs28364998 in SLC6A3; rs6275 in DRD2) and disorders of fluent speech in the Han Chinese, but the small sample size in this study calls for further investigation on a larger sample scale to confirm our findings.

Of the SNPs assessed, the highest association is observed between the $\mathrm{C}$ allele at rs6277 in the DRD2 gene and disorders of fluent speech in the Han Chinese who speak Mandarin. Significantly higher CC and lower CT genotype frequencies were observed in the patients compared with controls. The haplotype 939T/957T is a protective factor to the occurrence of speech disfluency. This study indicates that the higher susceptibility is because of the presence of the $\mathrm{C}$ allele as a risk factor and the loss of protective effect from the T allele at 6277 SNP. However, the real role of $D R D 2$ gene polymorphism in the pathogenesis of disorders of fluent speech should be further investigated by the combination of large population-based case-control studies and a functional analysis of the cellular signaling pathway of the DRD2.

\section{ACKNOWLEDGEMENTS}

This study was supported by the Dingguagua Stutter Club in Xi' an and China Stutter Association, and funded by the Major State Basic Research Program-973 of China (2005CB522804) and the Natural Science Foundation of Capital Medical University, Beijing (2008ZR05).

1 Saussure, F. D. Course in General Linguistics (P. Owen, London, 1960).

2 Dell, G. A spreading activation theory of retrieval in language production. Psychol. Rev. 93, 283-321 (1986).

3 Levelt, W. Models of word production. Trends. Cogn. Sci. 3, 223-232 (1999).

4 Bloodstein, O. A Handbook on Stuttering (Singular Pub. Group, San Diego, California, 1997).

5 Costa, D. \& Kroll, R. Stuttering: an update for physicians. Can. Med. Assoc. J. 162, 1849-1855 (2000).

6 Comings, D. E., Wu, S., Chiu, C., Ring, R. H., Gade, R., Ahn, C. et al. Polygenic inheritance of Tourette syndrome, stuttering, attention deficit hyperactivity, conduct, and oppositional defiant disorder: The additive and subtractive effect of the three dopaminergic genes-DRD2, D-beta-H, and DAT1. Am. J. Med. Genet. 67, 264-288 (1996).

7 Maguire, G., Riley, G., Franklin, D., Maguire, M., Nguyen, C. \& Brojeni, P. Olanzapine in the treatment of developmental stuttering: a double-blind, placebo-controlled trial. Ann. Clin. Psychiatry 16, 63-67 (2004).

8 Maguire, G. A., Yu, B. P., Franklin, D. L. \& Riley, G. D. Alleviating stuttering with pharmacological interventions. Expert Opin. Pharmacother. 5, 1565-1571 (2004).

9 Wu, J. C., Maguire, G., Riley, G., Lee, A., Keator, D., Tang, C. et al. Increased dopamine activity associated with stuttering. Neuroreport 8, 767-770 (1997).

10 Goberman, A. M. \& Blomgren, M. Parkinsonian speech disfluencies: effects of L-doparelated fluctuations. J. Fluency Disord. 28, 55-70 (2003).

11 Kent, L. The use of tranquilizers in the treatment of stuttering. J Speech Hear Disord 28, 288-294 (1963).

12 Maguire, G. A., Gottschalk, L. A., Riley, G. D., Franklin, D. L., Bechtel, R. J. \& Ashurst, J. Stuttering: Neuropsychiatric features measured by content analysis of speech and the effect of risperidone on stuttering severity. Compr. Psychiat. 40, 308-314 (1999).

13 Stager, S. V., Calis, K., Grothe, D., Bloch, M., Berensen, N. M., Smith, P. J. et al. Treatment with medications affecting dopaminergic and serotonergic mechanisms: Effects on fluency and anxiety in persons who stutter. J. Fluency Disord. 30, 319-335 (2005).

14 Maguire, G. A., Riley, G. D., Franklin, D. L. \& Gottschalk, L. A. Risperidone for the treatment of stuttering. J. Clin. Psychopharmacol. 20, 479-482 (2000).

15 Healey, E. C. \& Reid, R. ADHD and stuttering: a tutorial. J. Fluency Disord. 28, 79-93 (2003).

16 Mazei-Robison, M. S., Couch, R. S., Shelton, R. C., Stein, M. A. \& Blakely, R. D. Sequence variation in the human dopamine transporter gene in children with attention deficit hyperactivity disorder. Neuropharmacology 49, 724-736 (2005).

17 Bowton, E., Mazei-Robison, M., Blakely, R. \& Galli, A. Dopamine transporter coding variant Ala559Val associated with attention deficit hyperactivity disorder (ADHD) causes alteration of dopamine efflux. FENS Forum Abstracts 3, A060.4 (2006).

18 Rodriguez-Jimenez, R., Hoenicka, J., Jimenez-Arriero, M. A., Ponce, G., Bagney, A., Aragues, M. et al. Performance in the Wisconsin card sorting test and the C957T polymorphism of the DRD2 gene in healthy volunteers. Neuropsychobiology $54,166-$ 170 (2006).

19 Ryan, B. P. Programmed Therapy for Stuttering in Children and Adults (Thomas, Springfield, IL, 1974)

20 Shi, Y. Y. \& He, L. SHEsis, a powerful software platform for analyses of linkage disequilibrium, haplotype construction, and genetic association at polymorphism loci. Cell Res. 15, 97-98 (2005).

21 Bosshardt, $\mathrm{H}$. Effects of concurrent cognitive processing on the fluency of word repetition: comparison between persons who do and do not stutter. J. Fluency Disord. 27, 93-114 (2002).

22 Kellendonk, C., Simpson, E., Polan, H., Malleret, G., Vronskaya, S., Winiger, V. et al. Transient and selective overexpression of dopamine D2 receptors in the striatum causes persistent abnormalities in prefrontal cortex functioning. Neuron 49, 603-615 (2006).

23 Hirvonen, M., Laakso, A., Någren, K., Rinne, J., Pohjalainen, T. \& Hietala, J. C957T polymorphism of the dopamine D2 receptor (DRD2) gene affects striatal DRD2 availability in vivo. Mol. Psychiatr. 9, 1060-1061 (2004). 